\title{
A RECIPROCITY LAW FOR POLYNOMIALS WITH BERNOULLI COEFFICIENTS
}

BY

\author{
WILLEM FOUCHE
}

\begin{abstract}
We study the zeros $(\bmod p)$ of the polynomial $\beta_{p}(X)=$ $\sum_{k}\left(B_{k} / k\right)\left(X^{p-1-k}-1\right)$ for $p$ an odd prime, where $B_{k}$ denotes the $k$ th Bernoulli number and the summation extends over $1 \leqslant k \leqslant p-2$. We establish a reciprocity law which relates the congruence $\beta_{p}(r) \equiv 0(\bmod p)$ to a congruence $f_{p}(n) \equiv 0$ $(\bmod r)$ for $r$ a prime less than $p$ and $n \in \mathbf{Z}$. The polynomial $f_{p}(x)$ is the irreducible polynomial over $\mathbf{Q}$ of the number $\operatorname{Tr}_{l}^{\mathbf{Q}(\zeta)} \zeta$, where $\zeta$ is a primitive $p^{2}$ th root of unity and $L \subset \mathbf{Q}(\zeta)$ is the extension of degree $p$ over $\mathbf{Q}$. These congruences are closely related to the prime divisors of the indices $I(\alpha)=(\mathcal{O}: \mathbf{Z}[\alpha])$, where $\mathcal{O}$ is the integral closure in $L$ and $\alpha \in \mathcal{O}$ is of degree $p$ over $\mathbf{Q}$. We establish congruences $(\bmod p)$ involving the numbers $I(\alpha)$ and show that their prime divisors $r \neq p$ are closely related to the congruence $r^{p-1} \equiv 1\left(\bmod p^{2}\right)$.
\end{abstract}

0. Introduction. If the Bernoulli numbers $B_{k}, k=0,1, \ldots$, are given by the expansion

$$
\frac{t}{e^{t}-1}=\sum_{k=0}^{\infty} B_{k} \frac{t^{k}}{k !}
$$

then one defines, for $p$ an odd prime, the polynomial

$$
\beta_{p}(X)=\sum_{k=1}^{p-2} \frac{B_{k}}{k}\left(X^{p-1-k}-1\right) .
$$

Note that the coefficients of this polynomial are $p$-integral (Kummer).

In this paper we prove the equivalence of the congruence $\beta_{p}(r) \equiv 0(\bmod p)$, where $r$ is a prime such that $r<p$, to a polynomial congruence $(\bmod r)$. In order to construct these polynomial congruences, we introduce a class of cyclic extensions of Q.

Let $\zeta$ be a primitive $p^{2}$ th root of unity ( $p$ an odd prime). Then $\operatorname{Gal}(\mathbf{Q}(\zeta) / \mathbf{Q})$ contains a unique subgroup $H$ of order $p-1$. Let $L$ be the corresponding fixed field. We define

$$
H_{p}=\operatorname{Tr}_{L}^{\mathbf{Q}(\zeta) \zeta .}
$$

If one identifies $\operatorname{Gal}(\mathbf{Q}(\zeta) / \mathbf{Q})$ with $\left(\mathbf{Z} / p^{2} \mathbf{Z}\right)^{\times}$in the usual way, then one finds that $H \equiv\left\{\alpha^{p}\left(\bmod p^{2}\right): 1 \leqslant \alpha \leqslant p-1\right\}$. Hence

$$
H_{p}=\sum_{1 \leqslant \alpha \leqslant p-1} \zeta^{\alpha^{p}}
$$

Received by the editors July 5, 1983.

1980 Mathematics Subject Classification. Primary 12A35; Secondary 12A50.

Key words and phrases. Bernoulli numbers, Fermat quotients, discriminants, cyclic abelian extensions. 
Since Heilbronn raised the problem of finding nontrivial upper bounds for these trigonometric sums, we shall refer to them as Heilbronn sums [9, 2]. These sums are studied in [2] in connection with Fermat quotients; they are also closely related to certain $n$-dimensional Kloosterman sums (see, for example, [9, p. 342]).

Let $f_{p}(X)$ denote the irreducible polynomial of $H_{p}$ over $\mathbf{Q}$. A table of these polynomials and their discriminants $(p \leqslant 19)$ appears in [5, p. 292], where they are studied in connection with problems in cyclotomy.

It will be shown that if $p$ and $r$ are distinct primes with $p$ odd, then the congruence $f_{p}(n) \equiv 0(\bmod r)$ has an integral solution if and only if $\beta_{p}(r) \equiv r^{-1}[r / p](\bmod p)$. (If $y \in \mathbf{R}$, then $[y]$ is the largest integer $\leqslant y$.) We shall establish this result by showing that both these congruences hold if and only if $r^{p-1} \equiv 1\left(\bmod p^{2}\right)$ (Theorem 4.1).

Let $\mathcal{O}$ denote the ring of integers in $L$. If $\alpha \in \mathcal{O}-\mathbf{Z}$, then $\alpha$ is of degree $p$ over $\mathbf{Z}$; consequently, $\mathbf{Z}[\alpha]$ is a free abelian group of rank $p$ and the number $I(\alpha)=(O: \mathbf{Z}[\alpha])$ is well defined. We shall study the arithmetic properties of these numbers. In particular, it will be shown that

$$
I^{2}\left(H_{p}\right) \equiv(-1)^{(p+1) / 2} \quad\left(\bmod p^{2}\right) ;
$$

and if $p \equiv 1(\bmod 4)$, then $I(\alpha)>p$ for every $\alpha$ of degree $p$ over $\mathbf{Z}$. Finally, a prime $r$ divides $I(\alpha)$ for every $\alpha$ of degree $p$ over $\mathbf{Z}$ if and only if $r<p$ and $\beta_{p}(r) \equiv 0$ $(\bmod p)$.

1. Local results. In the sequel, $p$ will be an odd prime number, while $\mathbf{Q}_{p}$ will denote the field of $p$-adic numbers; $\mathbf{Z}_{p}$ is the ring of $p$-adic integers. If $A$ is a ring, we denote by $A^{\times}$the multiplicative group consisting of the units of $A$. If $L$ is a field which is a finite extension of $\mathbf{Q}_{p}$, then we write $N\left(L^{\times}\right)$for the image of $L^{\times}$under the norm map from $L$ to $\mathbf{Q}_{p}$. If $m \in \mathbf{Z}_{p}$, then $m^{\mathbf{Z}}$ stands for the multiplicative group consisting of the integral powers of $m$ and $\mu_{p-1}$ denotes the group consisting of the roots of unity in $\mathbf{Q}_{p}$. Finally, for $i=1,2, U_{i}$ is the subgroup $1+p^{i} \mathbf{Z}_{p}$ of $\mathbf{Z}_{p}^{\times}$.

It is well known that $\mathbf{Q}_{p}$ possesses exactly $p+1$ cyclic extensions of degree $p$. We shall need an explicit description of the norm groups of these extensions. With this object in mind, we introduce the following subgroups of $\mathbf{Q}_{p}^{\times}$: Let $u_{0}=1, u_{1}, \ldots, u_{p-1}$ be $p$ distinct representatives of the quotient group $U_{1} / U_{2}$. Then it is clear that $G_{i}=\left(u_{i} p\right)^{\mathbf{Z}} \cdot \mu_{p-1} \cdot U_{2}, i=0, \ldots, p-1$, and $G_{p}=p^{p \mathbf{Z}} \cdot \mu_{p-1} \cdot U_{1}$ are $p+1$ distinct open subgroups of $\mathbf{Q}_{p}^{\times}$of index $p$. These are the only subgroups of $\mathbf{Q}_{p}^{\times}$having this property: If $H$ is a subgroup of $\mathbf{Q}_{p}^{\times}$of index $p$, then, since $\mu_{p-1}$ is of order $p-1$ which is prime to $p$, the group $\mu_{p-1}$ is contained in $H$. Therefore, in order to count the subgroups of $\mathbf{Q}_{p}^{\times}$of index $p$, we need only consider the subgroups of $\mathbf{Q}_{p}^{\times} / \mu_{p-1}$ $\cong \mathbf{Z} \oplus \mathbf{Z}_{p}$; it is trivial that the latter group contains exactly $p+1$ subgroups of index $p$.

Let $\mathbf{C}_{p}$ denote an algebraic closure of $\mathbf{Q}_{p}$. Then, by local class field theory, the groups $G_{i}$ correspond to the cyclic extensions $L_{i}$ of $\mathbf{Q}_{p}$ in $\mathbf{C}_{p}$ of degree $p$ over $\mathbf{Q}_{p}$ in such a manner that for $i=0, \ldots, p$, we have $N\left(L_{i}^{\times}\right)=G_{i}$. In particular, $L_{0}$ is the unique subfield of $\mathbf{C}_{p}$ such that $L_{0}$ is a finite abelian extension of $\mathbf{Q}_{p}$ and

$$
N\left(L_{0}^{\times}\right)=p^{\mathbf{z}} \cdot \mu_{p-1} \cdot U_{2} .
$$


It we take the structure of the norm groups $G_{i}$ into account, we see that $L_{0}$ is the only extension $L$ of degree $p$ over $\mathbf{Q}_{p}$ such that for some element $\pi$ of $L$ it follows that $N \pi=p$.

Let $K$ be the extension in $\mathbf{C}_{p}$ of $\mathbf{Q}_{p}$ obtained by adjoining a primitive $p^{2}$ th root of unity $\zeta$ to $\mathbf{Q}_{p}$ and let $L^{\prime}$ be the subfield of $K$ of degree $p$ over $\mathbf{Q}_{p}$. If we write $\pi=N_{L^{\prime}}^{K}(\zeta-1)$, then $N_{\mathbf{Q}_{p}}^{L^{\prime}}(\pi)=N_{\mathbf{Q}_{p}}^{K}(\zeta-1)=p$. It follows from the remark in the preceding paragraph that $L^{\prime}=L_{0}$.

Let $\psi$ be a nontrivial character on $\mathbf{Q}_{p}^{\times} / N\left(L_{0}^{\times}\right)$; then it follows from (1.1) that $\psi$ has conductor $p^{2} \mathbf{Z}_{p}$. Since there are precisely $p-1$ nontrivial characters on $\mathbf{Q}_{p}^{\times} / N\left(L_{0}^{\times}\right)$, it follows from the conductor-discriminant formula (see e.g. [10, p. 240]) that

$$
d=p^{2(p-1)} \mathbf{Z}_{p}
$$

where $d$ denotes the discriminant of the extension $L_{0} / \mathbf{Q}_{\dot{p}}$. It is clear that $L_{0}$ is a fully ramified extension $\mathbf{Q}_{p}$; therefore

$$
D=\mathfrak{B}^{2(p-1)},
$$

where $D$ denotes the different of the extension and $\mathfrak{B}$ is the maximal ideal in the ring of integers $\mathcal{O}$ of $L_{0}$.

For every $\alpha \in L_{0}$, we denote by $\omega(\alpha)$ the order of $\alpha$ at $\mathfrak{B}$. If $\pi$ is of order 1 at $\mathfrak{P}$ and $f(X)$ is the irreducible polynomial of $\pi$ over $\mathbf{Q}_{p}$, then it follows from (1.3) that

$$
\omega\left(f^{\prime}(\pi)\right)=2(p-1) \text {. }
$$

On account of (1.1) and (1.3) we now have for a unique $\varepsilon \in \mu_{p-1}$ and a unique $a(\pi) \in \mathbf{Z}_{p}$ that

$$
N\left(f^{\prime}(\pi)\right)=\varepsilon\left(1+a(\pi) p^{2}\right) p^{2(p-1)} .
$$

We shall show in $\S 5$ that $\varepsilon=-1$.

Finally, we now show that for $\alpha \in L_{0}^{\times}$, we have

$$
\omega(\alpha-\sigma \alpha)>\omega(\alpha)
$$

for every $\sigma$ in the Galois group of the extension $L_{0}$ over $\mathbf{Q}_{p}$. Indeed, it follows from (1.1) that, if $x \in L_{0}^{\times}$is such that $N x=1$, then $x \in 1+\mathfrak{B}$. The result now follows if we put $x=\sigma \alpha / \alpha$.

2. Heilbronn sums. In the sequel, $\zeta, L, \mathcal{O}$ and $H_{p}$ will be as defined in the Introduction. Let $\mathfrak{B}$ be the prime ideal in $\mathcal{O}$ that lies above $p$. If $\alpha \in \mathcal{O}$, we denote by $\omega(\alpha)$ the order of $\alpha$ at $\mathfrak{B}$.

Note that, if we imbed $L$ in an algebraic closure of $\mathbf{Q}_{p}$, then the field $L_{0}$ given by (1.1) is the completion of $L$ at $\mathfrak{B}$. Consequently, the different $D$ of the extension $L / \mathbf{Q}$ is given by (1.3).

The following proposition deals with two rather special properties of Heilbronn sums:

Proposition 2.1. (a) $\omega\left(H_{p}+1\right)=1$.

(b) If $r$ is a rational prime such that $r \neq p$ and $R$ is a prime ideal in $\mathcal{O}$ that lies above $r$, then, for some $\sigma \in \operatorname{Gal}(L / Q)$, it follows that $\sigma H_{p} \not \equiv H_{p}(\bmod R)$. 
Proof. (a) Let $\pi=(\zeta-1)$ be the prime ideal in $\mathbf{Z}[\zeta]$ that lies above $p$. Then every $p^{2}$ th root of unity is $1(\bmod \pi)$ and $H_{p}$ is the sum of $p-1$ distinct $p^{2}$ th roots of unity (see (0.2)). Consequently

$$
\omega\left(H_{p}+1\right) \geqslant 1 .
$$

Since $\operatorname{Tr}_{\mathbf{Q}}^{L} D^{-1} \subseteq \mathbf{Z}$ and $p \mathcal{O}=\mathfrak{B}^{p}$, by $(1.3)$, we have $\operatorname{Tr}_{\mathbf{Q}}^{L}\left(D^{-1}\right)=\operatorname{Tr}_{\mathbf{Q}}^{L}\left(\mathfrak{H}^{-2 p} \cdot \mathfrak{P}^{2}\right)=$ $p^{-2} \operatorname{Tr}_{\mathbf{Q}}^{L}\left(\mathfrak{B}^{2}\right) \subseteq \mathbf{Z}$; in particular

$$
\operatorname{Tr}_{\mathbf{Q}}^{L}\left(\mathfrak{P}^{2}\right) \subseteq p^{2} \mathbf{Z}
$$

Suppose that $\omega\left(H_{p}+1\right)>1$, then, by $(2.2), \operatorname{Tr}_{\mathbf{Q}}^{L}\left(H_{p}+1\right) \equiv 0\left(\bmod p^{2}\right)$. On the other hand, $\operatorname{Tr}_{\mathbf{Q}}^{\mathbf{Q}(\zeta)}(\zeta)=0$ and it follows from $(0.1)$ that $\operatorname{Tr}_{\mathbf{Q}}^{L}\left(H_{p}+1\right)=p-\mathbf{a}$ contradiction. The result now follows from (2.1).

A proof of (b) appears in [2].

3. Bernoulli numbers and Fermat quotients. Let $p$ be an odd prime. For $x$ an integer such that $(x, p)=1$, let $q(x)$ denote the Fermat quotient $\left(x^{p-1}-1\right) / p$ $(\bmod p)$. It follows from the definition of $q(x)$ that

$$
q(x y) \equiv q(x)+q(y) \quad(\bmod p), \quad(x y, p)=1 .
$$

Proposition 3.1. Let $x$ be an integer such that $(x, p)=1$. Then

$$
q(x) \equiv \beta_{p}(x)-\frac{1}{x}\left[\frac{x}{p}\right](\bmod p) .
$$

Remark. Dickson [1, p. 112] attributes a formula similar to (3.2) to Nielsen (1915). The formula, as cited by Dickson, is incorrect, for the second term on the right-hand side of (3.2) is omitted. Since the original source is quite inaccessible (to the author), we have devised a proof of (3.2) in the formalism of $p$-adic measures as developed by Mazur [7].

Proof. We shall adhere to the notation and conventions of Koblitz [6, Chapter 2] in our application of $p$-adic measures in the sequel. The following is a brief summary of the results that will be needed: For every $x \in \mathbf{Z}$ which is prime to $p$, there exists a $\mathbf{Z}_{p}$-valued $p$-adic measure $\mu_{1, x}$ such that for $k \in \mathbf{Z}$, we have

$$
\begin{gathered}
\mu_{1, x}\left(k+p \mathbf{Z}_{p}\right)=\frac{1}{x}\left[\frac{k x}{p}\right]+\frac{1}{2}\left(\frac{1}{x}-1\right), \quad k \geqslant 0, \\
\int_{\mathbf{Z}_{p}} \alpha^{k-1} \mu_{1, x}=\left(1-x^{-k}\right) \frac{B_{k}}{k}, \quad k \geqslant 1,
\end{gathered}
$$

and

$$
\mu_{1, x}\left(\mathbf{Z}_{p}^{\times}\right)=0 .
$$

Here $\mathbf{Z}_{p}$ denotes the ring of $p$-adic integers, while $\mathbf{Z}_{p}^{\times}$is the group of units in $\mathbf{Z}_{p}$. Note that, since $\mu_{1 . x}$ is $\mathbf{Z}_{p}$-valued, for a $\mu_{1, x}$-integrable function $f$ on $\mathbf{Z}_{p}$, we have $\int_{\mathbf{z}_{p}} f \mu_{1, x} \equiv 0(\bmod p)$ whenever $f(x) \equiv 0(\bmod p)$ on $\mathbf{Z}_{p}$. By $(\mathrm{M} 2)$, we have

$$
\int_{\mathbf{Z}_{p}} \alpha^{p-2} \mu_{1, x}=\left(1-x^{-(p-1)}\right) B_{p-1} /(p-1) .
$$


Since $p B_{p-1} \equiv-1(\bmod p)$, on account of the von Staudt congruence, it follows that

$$
\int_{\mathbf{Z}_{p}} \alpha^{p-2} \mu_{1, x} \equiv\left(1-x^{-(p-1)}\right) / p \quad(\bmod p) .
$$

By (3.1), if $x^{-1}$ denotes the inverse of $x\left(\bmod p^{2}\right)$, then $q\left(x^{-1}\right) \equiv-q(x)(\bmod p)$. We have shown that

$$
q(x) \equiv \int_{\mathbf{Z}_{p}} \alpha^{p-2} \mu_{1, x}(\bmod p) .
$$

By (M3), we have for $p>3$

$$
\beta_{p}(x) \equiv \sum_{k=1}^{p-2} \frac{B_{k}}{k}\left(x^{-k}-1\right) \equiv B_{1}\left(x^{-1}-1\right)-\int_{\mathbf{z}_{p}}\left(\alpha+\cdots+\alpha^{p-3}\right) \mu_{1, x} \quad(\bmod p) .
$$

Since the integrand is $0(\bmod p)$ if $\alpha \in p \mathbf{Z}_{p}$ and $\mathbf{Z}_{p}^{\times}=\mathbf{Z}_{p}-p \mathbf{Z}_{p}$, we have, by (M3), that

$$
\beta_{p}(x) \equiv B_{1}\left(x^{-1}-1\right)-\int_{\mathbf{Z}_{p}^{\times}}\left(1+\alpha+\cdots+\alpha^{p-3}\right) \mu_{1, x} \quad(\bmod p) .
$$

By (3.3), the integral is congruent $(\bmod p)$ to

$$
\int_{\mathbf{Z}_{p}^{\times}}\left(1+\alpha+\cdots+\alpha^{p-2}\right) \mu_{1, x}-q(x) .
$$

If $\alpha \in \mathbf{Z}_{p}^{\times}-\left(1+p \mathbf{Z}_{p}\right)$, the integrand is equal to $\left(1-\alpha^{p-1}\right) /(1-\alpha) \equiv 0(\bmod p)$; hence (3.5) is congruent to

$$
\begin{aligned}
\int_{1+p \mathbf{Z}_{p}}(p-1) \mu_{1, x}-q(x) & \equiv-\mu_{1, x}\left(1+p \mathbf{Z}_{p}\right)-q(x) \\
& \equiv-\frac{1}{x}\left[\frac{x}{p}\right]-\frac{1}{2}\left(\frac{1}{x}-1\right)-q(x)
\end{aligned}
$$

in view of (M1) (with $k=1$ ). The result (3.2) (for $p>3$ ) now follows upon subtracting (3.6) from $B_{1}\left(x^{-1}-1\right)=-\left(x^{-1}-1\right) / 2$ in (3.4). The case $p=3$ is easily checked.

As an application of (3.2), we prove the following

Corollary. If $p \equiv 1(\bmod 4)$, then

$$
\sum_{2(\bmod 4)} \frac{B_{k}}{k}=\frac{B_{2}}{2}+\frac{B_{6}}{6}+\frac{B_{10}}{10}+\cdots+\frac{B_{p-3}}{p-3} \not \equiv 0 \quad(\bmod p)
$$

Proof. Let $n_{p}$ be an integer such that $n_{p}^{2} \equiv-1(\bmod p)$ and $1<n_{p}<p$. We shall prove

$$
\sum_{2(\bmod 4)} \frac{B_{k}}{k} \equiv \frac{1}{4}\left[\left(n_{p}+1\right)-\left(n_{p}^{2}+1\right) / p\right] \quad(\bmod p)
$$

It is easily seen that for $l \in \mathbf{Z}$, we have $q(-1+l p) \equiv l(\bmod p)$. Therefore, if we write $n_{p}^{2}=-1+l p$, we find that $q\left(n_{p}^{2}\right) \equiv l \equiv\left(n_{p}^{2}+1\right) / p(\bmod p)$. 
Hence, by (3.1), it follows that $q\left(n_{p}\right) \equiv\left(n_{p}^{2}+1\right) / 2 p$. On account of (3.2),

$$
\begin{aligned}
\left(n_{p}^{2}+1\right) / 2 p & \equiv \beta_{p}\left(n_{p}\right) \equiv \sum_{k=1}^{p} \frac{B_{k}}{k}\left(n_{p}^{-k}-1\right) \\
& \equiv \frac{1}{2}\left(n_{p}+1\right)+\sum_{k \geqslant 2} \frac{B_{k}}{k}\left(n_{p}^{-k}-1\right) \quad(\bmod p) .
\end{aligned}
$$

Since $B_{k}=0$ if $k \geqslant 3$ is odd, and $n_{p}^{-k} \equiv 1$ or $-1(\bmod p)$, according to whether $k \equiv 0$ or $2(\bmod 4)$, we see that $(3.8)$ holds.

Suppose now that (3.7) does not hold; then by (3.8), we have $n_{p}^{2}+1 \equiv p\left(n_{p}+1\right)$ $\left(\bmod p^{2}\right)$. Since both $n_{p}^{2}+1$ and $p\left(n_{p}+1\right)$ are positive and less than $p^{2}$, it must follow that $n_{p}^{2}+1=p\left(n_{p}+1\right)$ - which is impossible.

4. Reciprocity law. We shall prove the following

THEOREM 4.1. Let $r$ and $p$ be distinct primes with $p$ odd. Let $F$ denote the family of irreducible polynomials of elements in $\mathcal{O}$. Then the following statments are equivalent:

(a) $\beta_{p}(r) \equiv \frac{1}{r}\left[\frac{r}{p}\right](\bmod p)$.

(b) $f_{p}(n) \equiv 0(\bmod r)$ for some $n \in \mathbf{Z}$.

(c) For every $f(X) \in F$ there exists an integer $m$ such that $f(m) \equiv 0(\bmod r)$.

In the course of the proof of Theorem 4.1 we shall characterise the numbers $\alpha \in \mathcal{O}$ for which the theorem remains valid if we replace $f_{p}(X)$ in (b) by $\operatorname{Irr}(\alpha, L / \mathbf{Q}, X)$. If $r$ is a prime number and $R$ a prime ideal in $\mathcal{O}$ that divides $r$, we write $\overline{\mathcal{O}}_{R}=\mathcal{O} / R$ and $\overline{\mathbf{Z}}_{r}=\mathbf{Z} / r \mathbf{Z}$. If $\alpha \in \mathcal{O}$, we denote the image of $\alpha$ under the natural map $\mathcal{O} \rightarrow \overline{\mathcal{O}}_{R}$ by $\alpha_{R}$. Finally, we write $G=\operatorname{Gal}(L / \mathbf{Q})$.

Note that since $p$ is the only prime that ramifies in the extension $L / \mathbf{Q}$ and the extension is of prime degree, every prime number $r \neq p$ either splits completely in $\mathcal{O}$ or remains prime when lifted to $\mathcal{O}$.

LeMma 4.2. Let $r$ be a prime number $\neq p$. The congruences $f(x) \equiv 0(\bmod r)$, $f(X) \in F$, all have solutions in $\mathbf{Z}$ if and only if $r$ splits completely in $\mathcal{O}$.

Proof. Suppose $r$ does not split completely in $\mathcal{O}$. Then $R=r \mathcal{O}$ is prime and $\overline{\mathcal{O}}_{R}$ is a separable field extension of $\overline{\mathbf{Z}}_{r}$ of degree $p$. Choose $\mu$ in $\mathcal{O}$ such that $\mu_{R}$ generates $\overline{\mathcal{O}}_{R}$ over $\overline{\mathbf{Z}}_{r}$. Then, obviously, $\sigma \mu \not \equiv \mu(\bmod R)$ for at least one $\sigma \not \equiv$ id in $G$ and $\mu \not \equiv n$ $(\bmod R)$ for every $n \in \mathbf{Z}$; hence, the polynomial $\operatorname{Irr}(\mu, L / \mathbf{Q}, X)$ has no integral solutions modulo $r$. Conversely, if $r$ splits completely in $\mathcal{O}$, then $\overline{\mathcal{O}}_{R}=\overline{\mathbf{Z}}_{r}$ for every $R \mid r$ and the congruences all have solutions in $\mathbf{Z}$ modulo $r$.

Definition. Let $\alpha \in \mathcal{O}$. Then $\alpha$ is basic if, for every rational prime $r \neq p$ and prime divisor $R$ or $r$ in $\mathcal{O}$, it follows that $\overline{\mathcal{O}}_{R}=\overline{\mathbf{Z}}_{r}\left[\alpha_{R}\right]$.

REMARK. It is clear that if $\alpha$ generates a power basis of $\mathcal{O}$ over $\mathbf{Z}$, then $\alpha$ is basic. However, this is not a fruitful approach to the construction of examples of basic numbers since it will be showin in $\S 5$ that $\mathcal{O}$ has no power basis whenever $p \equiv 1$ $(\bmod 4)$. (On the other hand, if $p=3$, then $\mathcal{O}=\mathbf{Z}\left[H_{3}\right]$ !)

LEMMA 4.3. If $\alpha \in \mathcal{O}$, then the following statements are equivalent:

(i) $\alpha$ is basic. 
(ii) If $r(\neq p)$ is prime and $\operatorname{Irr}(\alpha, L / \mathbf{Q}, n) \equiv 0(\bmod r)$ for some $n \in \mathbf{Z}$, then the congruences $f(x) \equiv 0(\bmod r), f(X) \in F$ all have solutions in $\mathbf{Z}$.

(iii) If $r(\neq p), R \mid r$ and $\alpha \equiv n(\bmod R)$ for some $n \in \mathbf{Z}$, then $r$ splits completely in $\mathcal{O}$.

(iv) If the prime $r(\neq p)$ does not split in $\mathcal{O}$, then, for some $\sigma \in G$, it follows that $\sigma \alpha \not \equiv \alpha(\bmod r \mathcal{O})$.

Proof. It is clear from Lemma 4.2 that (ii) and (iii) are equivalent. We shall prove (i) $\Rightarrow$ (iii) $\Rightarrow$ (iv) $\Rightarrow$ (i).

(i) $\Rightarrow$ (iii): If (i) holds and $\alpha \equiv n(\bmod R)$, then $\overline{\mathcal{O}}_{R}=\overline{\mathbf{Z}}_{r}\left[\alpha_{R}\right]=\overline{\mathbf{Z}}_{r}$; hence the residue class degree of $R$ over $r$ is 1 and $r$ splits completely in $\mathcal{O}$.

(iii) $\Rightarrow$ (iv): Suppose $r$ does not split in $\mathcal{O}$ and $r \neq p$. Then $R=r \mathcal{O}$ is prime; if $\sigma \alpha \equiv \alpha(\bmod R)$ for every $\sigma \in G$, then for some $n \in \mathbf{Z}$ we have $\alpha \equiv n(\bmod R)$, in contradiction to (iii).

(iv) $\Rightarrow$ (i): If $r$ splits completely in $\mathcal{O}$, then $\alpha_{R} \in \overline{\mathbf{Z}}_{r}$ and $\overline{\mathcal{O}}_{R}=\overline{\mathbf{Z}}_{r}=\overline{\mathbf{Z}}_{r}\left[\alpha_{R}\right]$ for every $R \mid r$. Suppose that $r$ does not split completely in $\mathcal{O}$. Then $R=r \mathcal{O}$ is prime and by (iv), we have $\alpha_{R} \notin \overline{\mathbf{Z}}_{r}$. Since $\overline{\mathcal{O}}_{R} / \overline{\mathbf{Z}}_{r}$ is a field extension of prime degree, we have that $\overline{\mathcal{O}}_{R}=\overline{\mathbf{Z}}_{r}\left[\alpha_{R}\right]$.

LEMMA 4.4. The rational prime $r$ splits completely in $\mathcal{O}$ if and only if $r^{p-1} \equiv 1$ $\left(\bmod p^{2}\right)$.

Proof. This is immediate from the factorisation properties of rational primes when lifted to $\mathbf{Q}(\zeta)$.

Proof of Theorem 4.1. It follows from Proposition 2.1(b) and Lemma 4.3(iv) that $H_{p}$ is basic; hence (b) and (c) are equivalent, on account of Lemma 4.3(ii). By Lemma 4.2, Lemma 4.4 and Proposition 3.1 statements (a) and (c) hold if and only if $r^{p-1} \equiv 1\left(\bmod p^{2}\right)$. The proof is complete.

REMARKS. 1. In [2] it is shown that $q(2) \equiv 0(\bmod p)$ if and only if $f_{p}(0)=-N H_{p}$ is even, $N$ being the norm from $L$ to $\mathbf{Q}$. Consequently, the following statements are equivalent:

(i) $2^{p-1} \equiv 1\left(\bmod p^{2}\right)$;

(ii) $\mathrm{NH}_{p}$ is even;

(iii) $\beta_{p}(2) \equiv 0(\bmod p)$.

2. D. H. and E. Lehmer [5] show that if $f_{p}(n) \equiv 0(\bmod r)$ for some $n \in \mathbf{Z}$, then $q(r) \equiv 0(\bmod p)$. By Lemmas 4.3 and 4.4 this provides an alternative proof of the fact that $H_{p}$ is basic. They raised the question whether $f_{p}(X)$ could ever assume even values. We see from Remark 1 that it may happen and if it does, then $f_{p}(n)$ will be even when $n \equiv 0(\bmod p)$; this happens for $p \leqslant 6.10^{9}$ exactly when $p=1093$ or $p=3511[4]$.

5. Discriminants. If $\alpha \in \mathcal{O}$, we denote by $d(\alpha)$ the discriminant of the irreducible polynomial of $\alpha$ over $\mathbf{Q}$. Since $L$ is a totally real field, the absolute discriminant $d_{L}$ of the extension $L / Q$ is positive; hence, by (1.2), $d_{L}=p^{2(p-1)}$. Consequently, if $\alpha \in \mathcal{O}-\mathbf{Z}$, and $I(\alpha)=(\mathcal{O}: \mathbf{Z}[\alpha])$, then

$$
d(\alpha)=I^{2}(\alpha) p^{2(p-1)} .
$$


Furthermore, if $\alpha \in \mathcal{O}$, then

$$
d(\alpha)=(-1)^{p(p-1) / 2} N_{\mathbf{Q}}^{I}\left(f^{\prime}(\alpha)\right)
$$

where $f(X)=\operatorname{Irr}(\alpha, L / \mathbf{Q}, X)$.

It is shown in [5] that if $r(\neq p)$ is a prime divisor of $d\left(H_{p}\right)$, then $r^{p-1} \equiv 1$ $\left(\bmod p^{2}\right)$. It will be shown that the converse also holds, provided $r<p$. Indeed, we shall prove

Proposition 5.1. (a) If $\alpha \in \mathcal{O}$ is basic, then for every prime divisor $r \neq p$ of $d(\alpha)$, we have $r^{p-1} \equiv 1\left(\bmod p^{2}\right)$.

(b) If $r$ is a prime number, then $r \mid I(\alpha)$ for every $\alpha \in \mathcal{O}-\mathbf{Z}$ if and only if $r<p$ and $r^{p-1} \equiv 1\left(\bmod p^{2}\right)$.

Proof. (a) Let $G=\operatorname{Gal}(L / \mathbf{Q})$. If $r \mid d(\alpha)$ and $R \mid r$ in $\mathcal{O}$, then for some $\sigma, \tau \in G$ such that $\sigma \neq \tau$ we have $\sigma \alpha \equiv \tau \alpha(\bmod R)$. Suppose that $r \neq p$ and $r^{p-1} \not \equiv 1$ $\left(\bmod p^{2}\right)$. Then, by Lemma 4.4 , we have that $R=r \mathcal{O}$ is prime. In particular, $R$ remains invariant under the action of $G$. Hence $\tau^{-1} \sigma \alpha \equiv \alpha(\bmod R)$. Since $G$ is cyclic and of prime degree, $\sigma \alpha \equiv \alpha(\bmod R)$ for every $\sigma \in G$, in contradiction to Lemma 4.3(iv).

(b) Let $r<p$ be such that $r^{p-1} \equiv 1\left(\bmod p^{2}\right)$. Then, by Lemma $4.4, r$ splits completely in $\mathcal{O}$. Let $R \mid r$ in $\mathcal{O}$; then $\mathcal{O} / R=\mathbf{Z} / r \mathbf{Z}$ possesses $r$ distinct residue classes, i.e. $\alpha(\bmod R)$ can assume at most $r$ distinct values. On the other hand $G$ possesses $p>r$ elements. On account of Dirichlet's Box Principle, we conclude that for some $\sigma, \tau \in G$ such that $\sigma \neq \tau$, we have $\sigma \alpha \equiv \tau \alpha(\bmod R)$. Hence $r \mid d(\alpha)$ and $r \mid I(\alpha)$ by $(5.1)$.

Conversely, if $r \mid I(\alpha)$ for every $\alpha \in \mathcal{O}-\mathbf{Z}$, then $r \mid I\left(H_{p}\right)$ so that by (5.1) and (a) we have $r^{p-1} \equiv 1\left(\bmod p^{2}\right)$. Finally, it follows from Hensel's theory of indices of numbers fields [3] that if $r \mid I(\alpha)$ for every $\alpha \in \mathcal{O}-\mathbf{Z}$, then $r$ cannot exceed $p-1, p$ being the degree of the extension $L / \mathbf{Q}$ (see [8, Proposition 4.13, p. 165]).

The observations of $\S 1$ will enable us to prove the following

THEOREM 5.2 (a) If $\pi \in \mathcal{O}$ is of order 1 at the prime ideal above $p$, then

$$
I^{2}(\pi) \equiv(-1)^{(p+1) / 2} \quad\left(\bmod p^{2}\right) .
$$

(b) If $p \equiv 1(\bmod 4)$, then $I(\alpha)>p$ for every $\alpha \in \mathcal{O}-\mathbf{Z}$.

Remark. Note that (0.3) now follows from (a), Proposition 2.1(a) and the observation that $I\left(H_{p}+1\right)=I\left(H_{p}\right)$.

Proof. (a) As a first step we show, in the notation of $\$ 1$, that $\varepsilon=-1$ in (1.5). Let $\pi \in L_{0}$ be such that $\omega(\pi)=1$ and let $f(X)$ denote the irreducible polynomial of $\pi$ over $\mathbf{Q}_{p}$. Write $G=\operatorname{Gal}\left(L_{0} / \mathbf{Q}_{p}\right)$. Since $f^{\prime}(\pi)=\prod_{\sigma \neq \text { id }}(\pi-\sigma \pi)$, it follows from (1.4) that $\sum_{\sigma \neq i \mathrm{id}} \omega(\pi-\sigma \pi)=2(p-1)$. If we now take (1.6) into account, we find for every $\sigma \in G$ satisfying $\sigma \neq \mathrm{id}$, that

$$
\omega(\pi-\sigma \pi)=2 \text {. }
$$

For the remainder of the proof, $\sigma$ will denote a fixed generator of $G$. We define the sequence $v_{1}, v_{2}, \ldots$ by the formula $\sigma^{k} \pi=v_{k} \pi, k \geqslant 1$. By (5.3), we have for some 
$a \in \mathbf{Z}_{p}^{\times}$that $v_{1} \equiv 1+a \pi\left(\bmod \mathfrak{P}^{2}\right)$. We prove inductively that

$$
v_{k} \equiv(1+k a \pi) \quad\left(\bmod \mathfrak{B}^{2}\right), \quad k \geqslant 1 .
$$

Suppose that (5.4) holds for $k=l, l \geqslant 1$. Since $\sigma^{l+1} \pi=v_{l+1} \pi$ and $\sigma^{l+1} \pi=\sigma(v, \pi)$ $=\left(\sigma v_{l}\right) v_{1} \pi$, we have

$$
v_{l+1}=v_{1}\left(\sigma v_{l}\right) \equiv(1+a \pi)(1+l a \sigma \pi) \equiv 1+(l+1) a \pi \quad\left(\bmod \mathfrak{B}^{2}\right) ;
$$

the proof of (5.4) is complete. Since $G$ is cyclic and of order $p$,

$$
\begin{aligned}
f^{\prime}(\pi) & =\prod_{k=1}^{p-1}\left(\pi-\sigma^{k} \pi\right)=\left\{\prod_{k}\left(1-v_{k}\right)\right\} \pi^{p-1}=\left\{\prod_{k}\left(-k a \pi+O\left(\pi^{2}\right)\right)\right\} \pi^{p-1} \\
& =(-1)^{p-1}(p-1) ! a^{p-1} \pi^{2(p-1)}+O\left(\pi^{2 p-1}\right)=(-1) \pi^{2(p-1)}+O\left(\pi^{2 p-1}\right),
\end{aligned}
$$

where for $k \geqslant 1$ the symbol $O\left(\pi^{k}\right)$ stands for an element in $\mathfrak{B}^{k}$. Consequently, $f^{\prime}(\pi) / \pi^{2(p-1)} \equiv-1(\bmod \mathfrak{B})$. Since $N(-1+\mathfrak{B}) \subset-1+p \mathbf{Z}_{p}$ and $N\left(\pi^{2(p-1)}\right) \equiv$ $p^{2(p-1)}\left(\bmod p^{2 p-1}\right)$ it follows that

$$
N\left(f^{\prime}(\pi)\right) / p^{2(p-1)}=-1 \quad(\bmod p) .
$$

By (1.5), the same congruence holds with $\varepsilon$ in the place of -1 on the right-hand side of (5.5). Since the elements of $\mu_{p-1}$ are pairwise incongruent modulo $p$, we see that $\varepsilon=-1$. Consequently, if we imbed $L$ into $L_{0}$, we see that the congruence (5.5) holds modulo $p^{2}$ in $\mathbf{Z}$ provided $\pi$ lies in $L$. The proof of (a) is complete in view of (5.1) and (5.2).

(b) Let $\alpha \in \mathcal{O}-\mathbf{Z}$. Since $\mathcal{O} / \mathfrak{P}=\mathbf{Z} / p \mathbf{Z}$ and $\mathbf{Z}[\alpha+n]=\mathbf{Z}[\alpha]$ for every $n \in \mathbf{Z}$, we may assume that $\omega(\alpha) \geqslant 1$. If $p \equiv 1(\bmod 4)$ and $\omega(\alpha)=1$, it follows from (a) that $I^{2}(\alpha) \equiv-1\left(\bmod p^{2}\right)$. In particular, $I(\alpha) \geqslant l$, where $l$ is the smallest positive number such that $l^{2} \equiv-1\left(\bmod p^{2}\right)$; it is trivial that $l>p$. If $\omega(\alpha)>1$, then, by (1.6) we have for every $\sigma \in G$ that $\omega(\alpha-\sigma \alpha) \geqslant 3$. Let $f(X)$ denote the irreducible polynomial of $\alpha$ over $\mathbf{Q}$. Then $\omega\left(f^{\prime}(\alpha)\right) \geqslant 3(p-1)$; consequently, $d(\alpha) \equiv 0$ $\left(\bmod p^{3(p-1)}\right)$. By $(5.1)$, we find that $I(\alpha)$ is divisible by $p^{k}, k=(p-1) / 2$. In particular, $I(\alpha)>p$.

\section{REFERENCES}

1. L. E. Dickson, History of the theory of numbers: Vol. 1, Stechert, New York, 1934.

2. W. L. Fouché, Arithmetic properties of Heilbronn sums, J. Number Theory 19 (1984), 1-6.

3. K. Hensel, Arithmetische Untersuchungen über die gemeinsamen ausserwesentlichen Discriminantentheiler einer Gattung, J. Reine Angew. Math. 113 (1894), 128-160.

4. D. H. Lehmer, On Fermat's quotient, base 2, Math. Comp. 36 (1981), 289-290.

5. D. H. Lehmer and E. Lehmer, Cyclotomy for non-squarefree moduli, Proc. Analytic Number Theory (Philadelphia, 1980), Lecture Notes in Math., vol. 899, Springer-Verlag, Berlin and New York, 1981, pp. 276-300.

6. N. Koblitz, p-adic numbers, p-adic analysis, and zeta-functions, Springer-Verlag, Berlin and New York, 1977.

7. B. Mazur, Analyse p-adique, Bourbaki report, 1972.

8. W. Narkiewicz, Elementary and analytic theory of algebraic numbers, Polish Scientific Publishers. Warsaw, 1973.

9. R. A. Smith, On n-dimensional Kloosterman sums, J. Number Theory 11 (1979), 324-343.

10. A. Weil, Basic number theory, 3rd ed., Springer-Verlag, Berlin and New York, 1974.

Department of Mathematics, University of the Orange free State, Bloemfontein 9300. SOUTH AFrica 